\title{
Evaluating Nelipepimut-S in the Treatment of Breast Cancer: A Short Report on the Emerging Data
}

This article was published in the following Dove Press journal: Breast Cancer: Targets and Therapy

\author{
Patrick M Dillon (D) \\ Christiana M Brenin (D) \\ Craig L Slingluff Jr (D) ${ }^{2}$ \\ 'University of Virginia, Division of \\ Hematology/Oncology, Charlottesville, \\ VA 22908, USA; '2University of Virginia, \\ Department of Surgery, Charlottesville, \\ VA 22908, USA
}

\begin{abstract}
Vaccine therapies for treatment and prevention of cancer have seen modest degrees of efficacy with wide variation related to the tumor type, vaccine type, adjuvants and clinical setting for their study. Over the course of the last two decades, various peptide vaccines for breast cancer have been studied. The current leading peptide vaccine for human application is a HER2-based vaccine known as Nelipepimut-S, which has demonstrated immune activity and promising clinical activity in some settings. This review covers the development of this newer peptide vaccine for both HER2 amplified and non-amplified breast cancer.
\end{abstract}

Keywords: vaccine, breast cancer, HER2, peptide, human

\section{Introduction}

The human epidermal growth factor receptor 2 (HER2) protein has been one of the most extensively studied and targeted markers in oncology in the last 30 years. The identification of the HER2 receptor and the development of antibodies to target it such as trastuzumab, pertuzumab and others, are among the most successful advances in the treatment of breast cancer in the past 50 years. The research community learned that HER2 signalling through its membrane-bound tyrosine kinase domain results in downstream activation of a cascade of events leading to angiogenesis, cellular invasiveness, proliferation and survival proficiency. It is well known that about 20 percent of breast cancers will have marked overexpression of the HER2 receptor and will benefit from HER2 targeting agents. It is generally accepted that most of the other $80 \%$ of breast cancers will express HER2, but at lower levels. There remains debate about the potential role of the HER2 protein and HER2 targeting in lower expressing breast cancers. Nevertheless, as a target for either passive or active immunotherapy, HER2 has been immunogenic due to antigens such as HER $2_{369-377}$ (also known as the E75 peptide) that are easily recognized by $\mathrm{T}$ cells and dendritic cells.

The currently available agents approved for HER2-overexpressing breast cancer include: trastuzumab, ado-trastuzumab, pertuzumab, lapatinib, neratinib, most recently trastuzumab deruxtecan, and five trastuzumab biosimilars (as of 1/2020). Additionally, the novel HER2 targeted monoclonal antibody, margetuximab, and a small molecule inhibitor, tucatinib, are currently being reviewed by the US FDA for possible approvals. While some of those drugs have been tested in HER2-low
Correspondence: Patrick M Dillon Division of Hematology/Oncology, University of Virginia, Box 8007I6, Charlottesville, VA 22908, USA

Tel + I-434-982- | 495

Fax + I-434-244-7534

Email Pmd5b@hscmail.mcc.virginia.edu
Breast Cancer: Targets and Therapy 2020:12 69-75

69

(c) (i) (5) 2020 Dillon et al. This work is published and licensed by Dove Medical Press Limited. The full terms of this license are available at https://www.dovepress.com/terms.

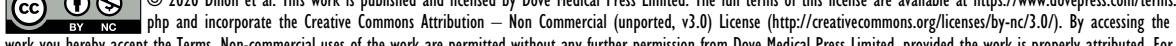
permission for commercial use of this work, please see paragraphs 4.2 and 5 of our Terms (https://www.dovepress.com/terms php). 
settings and non-breast settings, none have been approved to date for an indication outside of HER2-high or HER2 over-expressed cancer. Likewise, vaccine strategies have tested peptides, whole cell vaccines, dendritic cell vaccines, DNA vaccines and multipeptide vaccine in both HER2-high and HER2-low settings. The present review will examine the activity, development, efficacy and safety of the E75 peptide (also known as Nelipepimut-S when combined with GMCSF) as a peptide vaccine for breast cancer. Nelipepimut-S is currently in Phase III clinical development (NCT01479244) and has strong evidence of immunologic activity, though there is mixed evidence to date of clinical activity against early stage HER2overexpressed breast cancer and there is little clinical activity reported against advanced metastatic disease. There is emerging data on Nelipepimut-S for HER2-low and triple negative breast cancer that will be reviewed. ${ }^{1}$

\section{Methods: Literature Search, Inclusion and Exclusion Criteria}

We performed a systematic search of peer-reviewed literature databases from $11 / 1 / 2019$ to $12 / 9 / 2019$. This review was limited to manuscripts, abstracts and chapters available in the English language and catalogued in Pubmed, Web of Science, Scopus and proceedings of national meetings including: ASCO, SITC, SABCS, ESMO (American Society of Clinical Oncology, Society for ImmunoTherapy of Cancer, San Antonio Breast Cancer Symposium, and European Society of Medical Oncology). We searched for keywords including: "HER2 peptide" "E75 peptide", "Nelipepimut-S", "Neu-vax", "breast cancer". We excluded trials examining cancers other than breast cancer and other related peptides outside the studied amino acid sequence from HER2 $2_{369-377}$. Multipeptide vaccine studies were included for completeness.

\section{Background of Nelipepimut-S}

The aim of a cancer vaccine is to stimulate a cancer patient's immune system to recognize tumor associated antigens via active immunotherapy. Successful active immunotherapy results in $\mathrm{T}$ cell recognition and killing of cells expressing the antigen of interest. Ideally, successful $\mathrm{T}$ cell mediated tumor killing should lead to epitope spreading to increase the repertoire of $\mathrm{T}$ cells for cytolysis, and lead to long term $\mathrm{T}$ cell memory. Several peptide vaccines have been investigated for these purposes, and a peptide sequence that is successful to date is the E75 HER2 peptide vaccine, otherwise known as Nelipepimut-S. ${ }^{2}$ This vaccine has several published clinical and preclinical reports and has been studied in a registrational phase III study. ${ }^{3}$ Nelipepimut-S, or the E75 vaccine, is a 9 amino acid sequence from the extracellular domain of the HER2 receptor (residues 369-377 of HER2neu: KIFGSLAFL ${ }^{4}$ ). This 9 amino acid sequence has long been known to be the immunologically dominant epitope of the protein and is presented both by HLA-A2 and HLA-A3 (HLA restricted). ${ }^{2,5,6}$ These two alleles represent a majority of patients with breast cancer. HER2 is of course a self-antigen, but overexpression is largely limited to breast cancers (and occasionally lung, gastric and colon cancers). Surprisingly, there does not appear to be negative thymic selection of HER $2_{369-377}$ specific T cells as shown by several groups. ${ }^{7-10}$ Thus the E75 antigen from HER2 is a reasonable target for a variety of immunotherapies.

During in vitro preclinical development, the E75 peptide was recognized by CD8+ T lymphocytes. Subsequently, it was demonstrated that E75-stimulated cytotoxic $\mathrm{T}$ lymphocytes were capable of lysis of HER2-expressing cancer cell lines. ${ }^{4,10,11}$ The specificity of pulsed T cells for HER2 expressing cells was replicated in mouse models of cancer. ${ }^{8,12}$ Additionally, it was found by multiple groups that lymphocytes in circulation occasionally harbor pre-existing responses against the E75, 9 amino acid sequence HER2 neu $_{369-377}$ used in the subsequent development of Nelipepimut-S. ${ }^{6,11-13}$ Likewise, dendritic cells from normal donor blood sources have been shown to be able to present the E75 peptide and to generate E75-specific T cells. ${ }^{14}$

\section{Clinical Trials with the E75 Peptide}

The earliest Phase I pilot study of the E75 peptide in humans also incorporated a MUC1 peptide (M1.2) in an autologous dendritic cell vaccine in breast and ovarian cancer. ${ }^{14}$ Among 10 patients, CD8 responses to E75 and M1.2 were observed (via intracellular interferon assay and chromium release assays) in 5 patients. The authors also reported evidence of epitope spreading in two patients after repeat vaccination.

A study by Zaks and Rosenberg (Table 1) examined the activity of the single E75 peptide formulated with incomplete Freund's adjuvant in various solid tumors, including breast cancer. CD8 responses were again observed in human leukocyte antigen (HLA) -A2 and HLA-A3 patients, but anergy rather than memory was the long term outcome, ${ }^{15}$ possibly due to overstimulation related to incomplete Freund's adjuvant. 
Table I Ongoing or Completed Vaccine Trials with the HER2 ${ }_{369-377}$ Peptide and Associated Adjuvants Used and Systemic Therapies Combined with the Peptide

\begin{tabular}{|l|l|l|l|l|l|l|l|}
\hline $\begin{array}{l}\text { Trial } \\
\text { Number }\end{array}$ & Patients & $\begin{array}{l}\text { Vaccine } \\
\text { Antigen(s) }\end{array}$ & Adjuvant & $\begin{array}{l}\text { Systemic } \\
\text { Therapy }\end{array}$ & Ref & $\begin{array}{l}\text { Number of } \\
\text { participants }\end{array}$ & $\begin{array}{l}\text { First } \\
\text { Author }\end{array}$ \\
\hline NCT02636582 & DCIS & E75 & GM-CSF & None & {$[23]$} & 13 & Mittendorf \\
\hline NCT02297698 & HER2+ breast cancer & $\begin{array}{l}\text { E75 vs GM- } \\
\text { CSF }\end{array}$ & GM-CSF & Trastuzumab & {$[22]$} & 100 & Peace \\
\hline NCT01479244 & $\begin{array}{l}\text { Breast cancer with low- } \\
\text { intermediate HER2 }\end{array}$ & $\begin{array}{l}\text { E75 vs } \\
\text { placebo }\end{array}$ & GM-CSF & None & {$[3]$} & 758 & Mittendorf \\
\hline NCT0084I399 & Breast cancer & E75 & GM-CSF & None & {$[18-20]$} & 100 \\
\hline NCT00854789 & Breast cancer & E75 & GM-CSF & None & {$[17-19]$} & 95 & Mittendorf \\
\hline NCT01570036 & Breast cancer & E75 & GM-CSF & Trastuzumab & {$[1]$} & 275 & Mittendorf \\
\hline NCT00266II0 & Breast cancer & E75 & Dendritic & Trastuzumab + & {$[26]$} & 17 & Clifton \\
\hline Zaks '98 & $\begin{array}{l}\text { Breast, ovarian, and } \\
\text { colorectal cancer }\end{array}$ & E75 & $\begin{array}{l}\text { Incomplete } \\
\text { Freund's }\end{array}$ & None & {$[15]$} & 4 & Serody \\
\hline Knutson '02 & Breast/ovarian Cancer & E75 & GM-CSF & None & {$[16]$} & 6 \\
\hline Murray '02 & Breast/ovarian Cancer & E75 & GM-CSF & None & {$[17]$} & 14 & Zaks \\
\hline
\end{tabular}

Subsequently, the E75 peptide was combined with GMCSF to attempt to overcome the anergy suspected to be due to the incomplete Freund's adjuvant in the prior study. When combined with GM-CSF, the vaccine is termed NelipepimutS (also previously called Neu-vax). Two phase I studies of 6 and 14 patients respectively with advanced disease were completed using Nelipepimut-S by intradermal injection, and safety was observed for up to 1000 micrograms (mcg) of Nelipepimut-S along with $250 \mathrm{mcg}$ of GM-CSF. Immune response was observed by means of ELIspot and delayed type hypersensitivity analysis. ${ }^{16,17}$ Patients received monthly vaccinations for up to 10 months and no dose limiting toxicity was observed.

Given the challenge of developing an immunotherapy in heavily pre-treated metastatic patients, the Nelipepimut-S was subsequently tested in early stage, surgically resected breast cancer patients. Testing in early stage disease was expected to be safe given the excellent safety profile observed in the metastatic setting. Thus, a paired set of trials (NCT00841399, NCT00584789) were performed for stage II or stage III, HER2-expressing breast cancer as defined as any immunohistochemical (IHC) staining from $1+$ to $3+$. The sister clinical trials (phase II) were performed in the United States and have since been published. ${ }^{7,18,19}$ In the studies, all patients were clinically disease-free and were permitted to use concomitant endocrine therapies as well as prior Trastuzumab therapy. The dose and schedule were optimized in these early adjuvant trials. Ultimately, 195 patients were enrolled and followed for 60 months. ${ }^{18,19}$ There were 100 patients vaccinated and 95 control patients. In the primary analysis, with a median 20 months follow-up after vaccination, the recurrence rates were $5.6 \%$ vs $14.2 \%$ in vaccinated vs unvaccinated participants $(\mathrm{p}=0.04) .{ }^{20}$ Thus the studies met their primary endpoint. However, for secondary analysis it was found that the short-term recurrence difference observed at 20 months did not persist at any of the later analyses. For example, there was no difference in recurrence at the 26month analysis $(\mathrm{p}=0.15)$ nor at 60 months $(\mathrm{p}=0.08)$. There was no difference in overall survival (OS) with $p$ value $=0.1$. The authors suggested that waning immunity due to lack of boosting contributed to the lack of long term benefit of the vaccine strategy. ${ }^{20}$ Interestingly, there was a higher rate of visceral metastases in the vaccine-treated patients when they recurred. The toxicities of this vaccine strategy included flu-like symptoms, fatigue, and bone pain. Less than $2 \%$ of patients experienced any high grade toxicity (highest grade $=3$ ). The study concluded that an optimal dose would be $1000 \mathrm{mcg}$ and that a potential phase III trial should be performed. 
In the sister Phase II trials, boosting was explored and a suggestion of benefit was observed with boosting once every 6 months. Exploratory analysis also showed that the lowgrade breast cancers seemed to derive greater benefit from Nelipepimut-S vaccination than higher grade breast cancers. In a late followup report, there was only one recurrence observed in the 21 participants with optimal dosing of booster vaccines. ${ }^{18}$

Following completion of the phase II studies, a phase III study of Nelipepimut-S as a single agent, was designed and given the acronym, PRESENT. The PRESENT study (NCT01479244) fully accrued across 197 research sites by Galena Biopharma Inc. PRESENT was a registrational study of 758 patients with early stage, node positive breast cancer with low to intermediate HER2 expression with a primary endpoint of 3 year disease free survival (DFS). Patients received either Nelipepimut-S with GM-CSF or GM-CSF alone over the course of six intradermal injections followed by boosting every 6 months for 3 years. ${ }^{3}$ The interim analysis was published in 2019 and demonstrated no overall difference in disease free survival (DFS) between arms at 16 months follow-up. There was a numerically higher number of imaging-detected recurrences in the Nelipepimut-S vaccinated patients $(54.1 \%)$ compared to placebo $(29.2 \%){ }^{3}$ The phase III PRESENT study was discontinued due to futility in 2016, based on that interim analysis and the data monitoring committee recommendation. No new safety signals were reported, and no cardiac signals were seen. The protocol design required empiric cross-sectional body imaging yearly in all patients. This imaging requirement was a deviation from the existing standard of care (which would be for no cross sectional imaging unless symptoms arise). The required cross sectional imaging may have impacted on the early termination of the study. The control arm had a recurrence rate comparable to rates seen in contemporary trials in early stage patients. There is some speculation about whether pseudoprogression findings might have been observed in the radiographic recurrences, but biopsy confirmation was not undertaken, so no conclusion can be drawn. Given a negative phase III result in PRESENT, there is unclear future for the development of Nelipepimut-S in the node positive, HER2-low, adjuvant breast cancer population.

\section{Combination Clinical Trials with Nelipepimut-S}

Given the challenge and phase III disappointment of developing Nelipepimut-S as a stand-alone therapy, it is now also being examined in combinatorial studies. Preclinical data had suggested that trastuzumab could increase cross presentation of the E75 epitope and more efficient expansion of specific $\mathrm{CD}^{+} \mathrm{T}$ cells. The first phase IIb combination trial of E75 and trastuzumab (NCT01570036) enrolled 275 patients with HER2 low (IHC $1+$ or $2+$ ) breast cancer in the United States and combined Nelipepimut-S with trastuzumab. ${ }^{1}$ The patients received either Nelipepimut-S with trastuzumab or trastuzumab/GM-CSF. The study design was based on the observation, during the early phase Nelipepimut-S studies, that 12 patients were concurrently exposed to trastuzumab as a standard of care and none of those 12 patients experienced recurrent breast cancer. ${ }^{21}$ In this newer phase IIb combinatorial study of Nelipepimut-S with trastuzumab, overall, there was no statistically significant difference in DFS ( $p=0.18)$, although there was a benefit seen in the subgroup of patients deemed to be triple negative. In this subset of 97 patients, the DFS for Nelipepimut-S plus trastuzumab was $92.6 \%$ compared with $71.9 \%$ for the trastuzumab/GM-CSF group $(\mathrm{HR}=0.26, \mathrm{p}=0.01) .{ }^{1}$ This encouraging subset finding has reportedly led to the design of an upcoming clinical trial in the triple-negative early stage setting.

\section{Nelipepimut-S with Trastuzumab in HER2 IHC3+ Breast Cancer}

In a recently completed randomized phase II trial (NCT02297698), 100 patients with traditionally-defined HER2 overexpression (IHC $3+$ ) and otherwise high risk, non-metastatic breast cancer were enrolled to 1 to 1 randomized study of trastuzumab \pm Nelipepimut-S and followed for DFS. This study completed accrual in 2017, and interim results demonstrated that Nelipepimut-S was well tolerated, and no significant difference in side effect profile nor cardiac ejection fraction was observed between the two arms of the study. ${ }^{22}$ Clinical results have not been released to date.

\section{Trial of Nelipepimut-S in DCIS of the Breast}

A phase II study (NCT02636582) termed the VADIS study is assessing Nelipepimut-S against GM-CSF for ductal carcinoma in situ (DCIS) in the neo-adjuvant window of opportunity design. ${ }^{23}$ The premise for this is supported by work published by Lowenfeld et al. ${ }^{24}$ In this ongoing VADIS study, Nelipepimut-S or GM-CSF is given as two 
injections prior to definitive breast surgery. The primary endpoint is circulating immune response at 6 months after vaccination. Secondary endpoints are toxicity and safety. The study completed accrual in July 2019, and findings are still pending.

\section{Other E75 Studies in Breast Cancer}

The potential promise of Nelipepimut-S vaccines, but negative results in the large phase III trial, raise questions of whether alternate vaccine formulations may induce stronger and more effective immune responses. A recently published study created and tested a liposomal formulation of the vaccine by attaching the E75 peptide to the surface of distearoyl phosphocholine and distearoyl phophoglycerol of nano-liposomes for vaccination. ${ }^{25}$ ELISpot analysis and flow cytometry demonstrated significantly enhanced antitumor responses as well as tumor inhibition and prolonged survival time in the mouse TUBO model, which is a cell line that overexpresses the $\mathrm{rHER} / \mathrm{neu}$ protein. Thus, this approach offers promise for translation to human clinical trials. There is also an ongoing autologous dendritic cell vaccine of the E75 peptide in combination with vinorelbine and trastuzumab in human cancer patients at the University of North Carolina (NCT00266110). ${ }^{26}$ Finally, a series of four clinical trials performed at the University of Virginia incorporated the E75 peptide into multipeptide vaccines for breast and ovarian cancer, and using either polyICLC or incomplete Freund's adjuvant, rather than GM-CSF (NCT00892567, NCT00304096, NCT01532960, NCT00091273). Immune responses were detected, but clinical activity was not observed. $^{27,28}$

\section{Discussion}

The Nelipepimut-S vaccine alone demonstrates immune activity in patients expressing HLA-A2 or HLA-A3. As detailed above, the use of the Nelipepimut-S vaccine in adjuvant breast cancer settings has not led to clinically meaningful improvements in overall survival or disease free survival in a large randomized trial to date. Nevertheless, there are hints that this particular vaccine may hold potential clinical value in selected settings. For example, a meta-analysis of the 5 human clinical trials that involved randomization was performed in an effort to combine data from the smaller trials. In a published metaanalysis, the delayed hypersensitivity (DTH) responses and DFS combined data across trials suggested significant benefits to vaccination over control $(\mathrm{p}<0.05$ and $\mathrm{p}=0.001$ respectively). The combined data for OS and recurrence were suggested to also have relevance $(p=0.863$ and $p=-$ $0.388){ }^{29}$ The conclusions of the meta-analysis do notably differ from some of the individual trials and obviously the patient populations had major differences, thus rendering the impact of a meta-analysis unclear. Despite the criticisms of aggregation of data in the meta-analysis, it does suggest that in appropriate settings that the Nelipepimut-S may have clinical benefits for some patients without untoward toxicity. Raw data from the large 750 patient randomized phase III PRESENT trial, which was stopped for futility, was not available for analysis in that meta-analysis.

Thus, vaccine researchers in breast cancer are left wondering which direction to focus limited resources on. Clearly there is immunogenicity when vaccinating with the E75 peptide, and it tends to demonstrate synergy with passive antibody-based immunotherapy (ie, trastuzumab combinations). It is also intriguing that the triple negative early stage breast cancer population may have the greatest relative benefit after Nelipepimut-S vaccination. To date, there has been little traction in developing combinatorial strategies with checkpoint inhibitors or with myeloid suppressing immunotherapy strategies. With checkpoint inhibitors approved in the metastatic triple negative setting and expected in the triple negative adjuvant setting, it is unclear what role peptide vaccination strategies may be able to play in the future triple negative treatment landscape.

Some remaining concerns for the E75 HER2 peptide development include the criticism that the peptide is HLA restricted and thus not available universally to all patients. Also there is an unresolved question about how to address waning immunity and the need for long-term boosting strategies. Finally, the question about how best to select patients, especially in light of major immune system modulation that occurs during and immediately following adjuvant chemotherapy. It remains unknown whether the rebounding immune system in the 6 months following cytotoxic chemotherapy presents a stimulatory or suppressive environment for peptide vaccine generally and specifically for this Nelipepimut-S vaccine. Likewise, since HER2 targeting antibodies also impact on the immune recognition of antigens from HER2, it is further unclear whether early adjuvant vaccination at the time of adjuvant HER2 antibodies or following the course of HER2 maintenance antibodies will be optimal.

\section{Conclusion}

Nelipepimut-S demonstrated immune activity against HER2 positive breast cancer and suggestion of activity against triple 
negative breast cancer. Its development in the adjuvant HER2 low to intermediate population might be unlikely to continue based on the negative phase III PRESENT trial. Nevertheless, several important studies are yet to be performed for the Nelipepimut-S and related E75 vaccines, such as combinatorial studies, novel adjuvant studies, boosting strategies, and biomarker driven studies. Recently there is rising interest in vaccine therapy for breast cancer, so this or related vaccine strategies are likely to continue to be explored. Optimal patient selection and monitoring may aid in future development of this cancer therapy.

\section{Acknowledgments}

Drs. Dillon, Brenin and Slingluff are supported by NCI support grant: 2P30CA044579-26 for the University of Virginia Cancer Center.

\section{Disclosure}

Drs. Dillon and Slingluff have published studies on peptides referenced in this manuscript. The University of Virginia was a subsite for a clinical trial referenced in this manuscript. Dr Dillon participated in a clinical trial for Galena Pharmaceuticals. Dr. Slingluff is an inventor on licensed patents held by the University of Virginia Licensing and Ventures group for peptides used in melanoma vaccines. Dr Slingluff reports grants, non-financial support from Celldex for providing antibodies for clinical trials and for preclinical studies. He also reports grants and/or nonfinancial support from Merck, Immatics, Polynoma, and GlaxoSmithKline; non-financial support from Theraclion, outside the submitted work. Dr Slingluff also in the process of joining the scientific advisory board with CureVac. In addition, Dr Slingluff has patents on peptides used in cancer vaccines with royalties paid, a pending patent on biomarkers, a patent for a surgical device issued. The authors report no other conflicts of interest in this work.

\section{References}

1. Clifton GT, Hale D, Vreeland TJ. et al. Results of a randomized phase IIb trial of nelipepimut-S + trastuzumab vs trastuzumab to prevent recurrences in high-risk HER2 low-expressing breast cancer patients. Clin Cancer Res. 2020:clincanres.2741.2019. doi:10.1158/1078-0432. CCR-19-2741

2. Clifton GT, Peoples GE, Mittendorf EA. The development and use of the E75 (HER2 369-377) peptide vaccine. Future Oncol. 2016;12 (11):1321-1329. doi:10.2217/fon-2015-0054

3. Mittendorf EA, Lu B, Melisko M, et al. Efficacy and safety analysis of Nelipepimut-S vaccine to prevent breast cancer recurrence: a randomized, multicenter, phase III clinical trial. Clin Cancer Res. 2019;25(14):4248-4254. doi:10.1158/1078-0432.CCR-18-2867
4. Fisk B, Blevins TL, Wharton JT, Ioannides CG. Identification of an immunodominant peptide of HER-2/neu protooncogene recognized by ovarian tumor-specific cytotoxic T lymphocyte lines. $J$ Exp Med. 1995;181(6):2109-2117. doi:10.1084/jem.181.6.2109

5. Benavides LC, Gates JD, Carmichael MG, et al. The impact of HER2/neu expression level on response to the E75 vaccine: from U.S. Military Cancer Institute Clinical Trials Group Study I-01 and I-02. Clin Cancer Res. 2009;15(8):2895-2904. doi:10.1158/10780432.CCR-08-1126

6. Fisk B, Savary C, Hudson JM, et al. Changes in an HER-2 peptide upregulating HLA-A2 expression affect both conformational epitopes and CTL recognition: implications for optimization of antigen presentation and tumor-specific CTL induction. J Immunother Emphasis Tumor Immunol. 1995;18(4):197-209. doi:10.1097/00002371-19951100000001

7. Amin A, Benavides LC, Holmes JP, et al. Assessment of immunologic response and recurrence patterns among patients with clinical recurrence after vaccination with a preventive HER2/neu peptide vaccine: from US Military Cancer Institute Clinical Trials Group Study I-01 and I-02. Cancer Immunol Immunother. 2008;57 (12):1817-1825. doi:10.1007/s00262-008-0509-2

8. Brossart P, Stuhler G, Flad T, et al. Her-2/neu-derived peptides are tumor-associated antigens expressed by human renal cell and colon carcinoma lines and are recognized by in vitro induced specific cytotoxic T lymphocytes. Cancer Res. 1998;58(4):732-736.

9. Holmes JP, Clifton GT, Patil R, et al. Use of booster inoculations to sustain the clinical effect of an adjuvant breast cancer vaccine: from US Military Cancer Institute Clinical Trials Group Study I-01 and I-02. Cancer. 2011;117(3):463-471. doi:10.1002/cncr.25586

10. Kawashima I, Hudson SJ, Tsai V, et al. The multi-epitope approach for immunotherapy for cancer: identification of several CTL epitopes from various tumor-associated antigens expressed on solid epithelial tumors. Hum Immunol. 1998;59(1):1-14. doi:10.1016/S0198-8859(97)00255-3

11. Kono K, Takahashi A, Sugai H, et al. Dendritic cells pulsed with HER-2/neu-derived peptides can induce specific T-cell responses in patients with gastric cancer. Clin Cancer Res. 2002;8(11):3394-3400.

12. Lustgarten J, Theobald M, Labadie C, et al. Identification of Her-2/ Neu CTL epitopes using double transgenic mice expressing HLA-A2.1 and human CD.8. Hum Immunol. 1997;52(2):109-118. doi:10.1016/S0198-8859(96)00292-3

13. Kuerer HM, Peoples GE, Sahin AA, et al. Axillary lymph node cellular immune response to HER-2/neu peptides in patients with carcinoma of the breast. J Interferon Cytokine Res. 2002;22 (5):583-592. doi:10.1089/10799900252982061

14. Brossart P, Wirths S, Stuhler G, Reichardt VL, Kanz L, Brugger W. Induction of cytotoxic T-lymphocyte responses in vivo after vaccinations with peptide-pulsed dendritic cells. Blood. 2000;96 (9):3102-3108. doi:10.1182/blood.V96.9.3102

15. Zaks TZ, Rosenberg SA. Immunization with a peptide epitope (p369-377) from HER-2/neu leads to peptide-specific cytotoxic T lymphocytes that fail to recognize HER-2/neu+ tumors. Cancer Res. 1998;58(21):4902-4908.

16. Knutson KL, Schiffman K, Cheever MA, Disis ML. Immunization of cancer patients with a HER-2/neu, HLA-A2 peptide, p369-377, results in short-lived peptide-specific immunity. Clin Cancer Res. 2002;8(5):1014-1018.

17. Murray JL, Gillogly ME, Przepiorka D, et al. Toxicity, immunogenicity, and induction of E75-specific tumor-lytic CTLs by HER-2 peptide E75 (369-377) combined with granulocyte macrophage colony-stimulating factor in HLA-A2+ patients with metastatic breast and ovarian cancer. Clin Cancer Res. 2002;8(11):3407-3418.

18. Mittendorf EA, Clifton GT, Holmes JP, et al. Final report of the phase I/II clinical trial of the E75 (nelipepimut-S) vaccine with booster inoculations to prevent disease recurrence in high-risk breast cancer patients. Ann Oncol. 2014;25(9):1735-1742. doi:10.1093/annonc/ mdu 211 
19. Peoples GE, Gurney JM, Hueman MT, et al. Clinical trial results of a HER2/neu (E75) vaccine to prevent recurrence in high-risk breast cancer patients. J clin oncol. 2005;23(30):7536-7545. doi:10.1200/ JCO.2005.03.047

20. Peoples GE, Holmes JP, Hueman MT, et al. Combined clinical trial results of a HER2/neu (E75) vaccine for the prevention of recurrence in high-risk breast cancer patients: U.S. Military Cancer Institute Clinical Trials Group Study I-01 and I-02. Clin Cancer Res. 2008;14(3):797-803. doi:10.1158/1078-0432.CCR-07-1448

21. Mittendorf EA, Clifton GT, Holmes JP, et al. Clinical trial results of the HER-2/neu (E75) vaccine to prevent breast cancer recurrence in high-risk patients: from US Military Cancer Institute Clinical Trials Group Study I-01 and I-02. Cancer. 2012;118(10):2594-2602. doi:10.1002/cncr.26574

22. Peace KM, Litton JK, Murthy RK, et al. Pre-specified interim analysis in a prospective, randomized phase II trial of trastuzumab vs trastuzumab + NeuVax to prevent breast cancer recurrence in HER2 + breast cancer patients. Paper presented at: American Association of Cancer Researchers, Annual Meeting; 2017; Washington, DC.

23. Mittendorf Elizabeth A, Plitas G, Garber J, et al. Abstract OT3-0104: VADIS trial: phase II trial of the nelipepimut-S peptide vaccine in women with DCIS of the breast. Paper presented at: San Antonio Breast Cancer Symposium; 2016; San Antonio, TX.

24. Lowenfeld L, Mick R, Datta J, et al. Dendritic cell vaccination enhances immune responses and induces regression of HER2(pos) DCIS independent of route: results of randomized selection design trial. Clin Cancer Res. 2017;23(12):2961-2971. doi:10.1158/10780432.CCR-16-1924
25. Arab A, Behravan J, Razazan A, et al. A nano-liposome vaccine carrying E75, a HER-2/neu-derived peptide, exhibits significant antitumour activity in mice. J Drug Target. 2018;26(4):365-372. doi:10.1080/1061186X.2017.1387788

26. Serody J; 2020. Vaccine Therapy, Trastuzumab, and Vinorelbine in Treating Patients With Locally Recurrent or Metastatic Breast Cancer. Available from: https://clinicaltrials.gov/ct2/show/ NCT00266110. Accessed March 11, 2020.

27. Chianese-Bullock KA, Irvin WP Jr., Petroni GR, et al. A multipeptide vaccine is safe and elicits T-cell responses in participants with advanced stage ovarian cancer. J Immunother. 2008;31(4):420-430. doi:10.1097/CJI.0b013e31816dad10

28. Dillon PM, Petroni GR, Smolkin ME, et al. A pilot study of the immunogenicity of a 9-peptide breast cancer vaccine plus poly-ICLC in early stage breast cancer. J Immunother Cancer. 2017;5(1):92. doi:10.1186/s40425-017-0295-5

29. Chamani R, Ranji P, Hadji M, Nahvijou A, Esmati E, Alizadeh AM. Application of E75 peptide vaccine in breast cancer patients: a systematic review and meta-analysis. Eur $J$ Pharmacol. 2018;831:87-93. doi:10.1016/j.ejphar.2018.05.010

\section{Publish your work in this journal}

Breast Cancer - Targets and Therapy is an international, peer-reviewed open access journal focusing on breast cancer research, identification of therapeutic targets and the optimal use of preventative and integrated treatment interventions to achieve improved outcomes, enhanced survival and quality of life for the cancer patient.
The manuscript management system is completely online and includes a very quick and fair peer-review system, which is all easy to use. Visit http://www.dovepress.com/testimonials.php to read real quotes from published authors. 